\section{P186 The Pilot Light Summit: Development and Refinement of Consensus-Based Holistic Food Education Standards for Youth}

Jessica Metcalfe, MPH, jarick2@illinois.edu, University of Illinois, Urbana-Champaign, 904 W Nevada Street, Urbana, IL 61801; Carolyn Sutter, PhD; Alexandra DeSorbo-Quinn, EdD, MPH, Pilot Light; Lynn Tucker, MPH, RD, CDE; John Allegrante, PhD, MS, Teachers College, Columbia University; David Lohrmann, PhD, Indiana University, Bloomington; Pamela Koch, EdD, MS, MEd, Teachers College, Columbia University

Background (Background, Rationale, Prior Research, and/or Theory): Social Ecological Theory indicates that context at the micro and macro level plays an important role in our food choices. Therefore, holistic and context-focused food education can help children develop critical thinking skills that lead to healthy food choices in everyday life. Comprehensive food education programs should attend to the importance of nutrition to maintain good health and the role that food plays in our culture, relationships, history, and environment. In order to encourage the implementation of holistic food education, Pilot Light, a Chicago-based non-profit that provides K-12 teachers with food education curriculum and professional development, sought to develop clear Food Education Standards that support such holistic food education.

Objective: To convene a group of experts during the Pilot Light Summit to participate in the systematic development and refinement of Food Education Standards that guide the implementation of food education programs in schools.

Study Design, Setting, Participants, Intervention: The Pilot Light Food Education Summit convened 25 experts and community members across multiple disciplinary fields (including agriculture, culinary, education, nutrition, and public health) to develop and refine a consensus-based set of Food Education Standards and accompanying K-12 competencies that could be used to implement holistic food education in the classroom.

Outcome Measures and Analysis: A set of Food Education Standards, which included a list of 149 competencies in food education, were analyzed and categorized using thematic analysis.

Results: Thirty-one themes were derived from the original list of competencies; the most prevalent themes endorsed by Summit participants were food choice, health, food production, food access, and culture. Seven Food Education Standards were developed that encompassed all 31 themes.

Conclusions and Implications: The Food Education Standards developed at the Pilot Light Summit provide clear, accessible guidance for educators interested in incorporating holistic food education in the classroom. Pilot Light will incorporate Food Education Standards into its existing programming and encourage further dissemination. Funding: Pilot Light.
P187 The SWITCH Implementation Process on School Lunch Consumption Patterns and Plate Waste

Yuanying Lou, MS, Iowa State University; Huaiqing $W u$, PhD; Gregory J. Welk, PhD; Spyridoula Vazou, PhD; Senlin Chen, PhD, Louisiana State University; Douglas A. Gentile, PhD, Iowa State University; Ric Rosenkranz, PhD, Kansas State University; David A. Dzewaltowski, PhD, University of Nebraska Medical Center and Buffett Early Childhood Institute; Lorraine Lanningham-Foster, PhD, lmlf@iastate.edu, Iowa State University, 1117 Human Nutrition, 706 Morrill Road, Ames, IA 50011

Background (Background, Rationale, Prior Research, and/or Theory): Schools provide an ideal setting to promote positive lifestyle behaviors in youth. The SWITCH $^{\circledR}$ (School Wellness Integration Targeting Child Health) implementation process is designed to help schools operationalize and improve school wellness initiatives, including nutrition behaviors.

Objective: This study examined the influence of the SWITCH $^{\circledR}$ implementation process on school lunch consumption and waste, especially fruits and vegetables.

Study Design, Setting, Participants, Intervention: Four schools from a suburban school district in Iowa were included in the evaluation, two were participating in SWITCH $^{\circledR}$ and two were not. Before- and after-lunch photos of food trays were taken for every 5th-grade student participating in school lunch in both SWITCH ${ }^{\circledR}$ and control schools before SWITCH ${ }^{\circledR}$ started (baseline, 02/2017) and after SWITCH ${ }^{\circledR}$ finished (endpoint, 05/2017).

Outcome Measures and Analysis: Trained research assistants used the "Quarter System" to compare the beforeand-after- lunch photos for each person to estimate the waste percentage for individual food items on each tray. Based on the initial portion size, fruit and vegetable consumption was calculated. Linear mixed models were applied to analyze the effects of SWITCH ${ }^{\circledR}$, gender, and food types on the waste and consumption patterns.

Results: There was no significant decrease in overall school lunch waste (average percentage wasted across all food types) in both SWITCH ${ }^{\circledR}$ and control schools. And the school lunch waste was not significantly different between SWITCH ${ }^{\circledR}$ and control schools at either baseline or endpoint. However, students in SWITCH ${ }^{\circledR}$ schools significantly increased their fruit consumption $(P=.02)$ by increasing their initial portion size. Contrarily, the fruit consumption from baseline to endpoint decreased in control schools, although this decrease was not statistically significant. Boys consumed fewer vegetables than girls $(P<.0001)$. Although boys wasted fewer vegetables $(P<.0001)$, they selected much less to start $(P<.0001)$.

Conclusions and Implications: By increasing the selection portion size, SWITCH ${ }^{\circledR}$ implementations may have a positive influence on the fruits and vegetable consumption in children.

Funding: USDA. 


\section{P188 Thumbs Up for Healthy Choices: Making the Healthy Choice, the Easy Choice in Utah's Food Pantries}

Casey Coombs, RDN, casey.coombs@usu.edu, Utah SNAPEd, Utah State University, 8749 Old Main Hill, Logan, UT 84322; Mateja R. Savoie-Roskos, PhD, MPH, RDN, CNP, Utah State University; Heidi LeBlanc, MS, Utah SNAP-Ed, Utah State University

Background (Background, Rationale, Prior Research, and/or Theory): Food pantry users are often assumed to have poor diet quality. Identifying effective strategies that increase the selection of healthy foods by pantry users may help improve their dietary intake. Utah's Supplemental Nutrition Assistance Program-Education (SNAP-Ed) developed the Thumbs Up for Healthy Choices program which aims to make the healthy choice, the easy choice in pantries. Thumbs Up for Healthy Choices uses nudge strategies including shelf signs, recipe cards, and posters to increase the visibility of healthy options.

Objective: The objective of this study was to evaluate the effectiveness of the Thumbs Up for Healthy Choices program on food pantry users' selection and use of targeted foods.

Study Design, Setting, Participants, Intervention: A convenience sample of 457 food pantry users in six urban Utah food pantries were surveyed. Participants were asked about their familiarity with the Thumbs Up program, barriers to making healthy choices, selection of promoted items, use of foods and recipes at home, as well as the impact of the program on dietary intake.

Outcome Measures and Analysis: Frequencies and descriptive statistics were analyzed. Chi-square associations were used to identify relationships between demographic characteristics and program impact. Logistic regression was used to analyze the effect of exposure on program impacts.

Results: Eighty-four percent of respondents agree or strongly agree that Thumbs Up makes it easier to make healthy choices. Sixty-seven percent of respondents agree or strongly agree they eat healthier since Thumbs Up was implemented. Hispanics were more likely than nonHispanics to report healthier eating for themselves and their families $(P=.002, P=.001)$. After controlling for age, ethnicity, and gender, program exposure was significantly associated with using targeted foods at home $(P=<.001)$ and selecting foods with Thumbs Up signs $(P=.002)$.

Conclusions and Implications: These results suggest that the Thumbs Up for Healthy Choices program positively impacts food pantry users' selection of targeted healthy foods. Nudge programs, including Thumbs Up for Healthy Choices, are low-input strategies that may improve food pantry users' selection and consumption of healthy foods.

Funding: Supplemental Nutrition Assistance Program-Education.
P189 UC CalFresh Nutrition Education Program Empowering Low-Income Families with Knowledge and Skills About Food Resource Management

Deepa Srivastava,PhD, dsr@ucdavis.edu, University of California Cooperative Extension, 4437-B S Laspina Street, Tulare, CA 93274; Barbara Mknelly, MS

Objective: To improve food resource management behaviors essential to maximizing use of limited resources, supporting a healthy diet, and improving food security among low-income families.

Target Audience: Supplemental Nutrition Assistance Program Education (SNAP-Ed) eligible/ low-income adults/ families in Tulare County, California.

Theory, Prior Research, Rationale: The UC CalFresh NEP is guided by the SNAP-Ed Evaluation Framework.

Description: During the FFY2017, a four-week seriesbased Plan Shop Save Cook (PSSC) curriculum was administered to UC CalFresh program participants. The program included the following components: plan meals, use a shopping list, understand and use food labels, save money, and cook a meal.

Evaluation: A pre-post evaluation was conducted to determine behavioral change in planning meals, use of shopping list, use of food labels, saving money, and cooking a meal. Of the total participants $(n=98)$ who completed the pre-post survey, majority were Hispanic-Latino (94\%) and female (92\%). Paired sample t-test analysis indicated statistically significant results for the following behaviors: shop with a list (t $(97)=2.333, P=.022)$, think about health choices $(\mathrm{t}(97)=2.303, P=.023)$, plan meals $(\mathrm{t}$ $(97)=3.790, P=.000)$, use nutrition facts label $(\mathrm{t}$ $(97)=4.519, P=.000)$, use MyPlate $(\mathrm{t}(97)=6.764, P=.000)$, and all five behaviors ( $\mathrm{t}(97)=4.619, P=.000)$.

Conclusions and Implications: Through a series of nutrition classes, UC CalFresh NEP improved knowledge and skills of adult participants to plan tasty, cost-effective, and nutritious meals. Findings provide essential insight into behavioral strategies (e.g. shopping with a list, planning meals, reading nutrition facts labels) for communicating food resource management messages to low-income families. Nutrition education professionals can use these food resource management strategies to tailor educational messages for limited resources families.

Funding: California SNAP-Ed (UC CalFresh NEP).

\section{P190 Utilization of a Near-Peer Nutrition Education Program for Undergraduates}

Crystal C.Douglas,PhD, RDN, ccdouglas@shsu.edu, Sam Houston State University, 1700 University Avenue, Huntsville, TX 77341; Simone P. Camel, PhD, RDN

Objective: Literature supports the use of peer-mentors to provide education and guidance for improving health behaviors; however, there is a paucity of research specifically utilizing motivational interviewing and counseling 